Received: May 22, 2017

Accepted: June 02, 2017

Published: June 12, 2017

\title{
Actinic Keratosis - the Probable Cause of Squamous Cell Skin Carcinomas
}

\author{
Latha Uppala* \\ Department of Pharmaceutics, SSJ College of Pharmacy, Hyderabad, India \\ *Corresponding author: Latha Uppala, Department of Pharmaceutics, SSJ College of Pharmacy, \\ Hyderabad, India, E-mail: chowdarylatha79@gmail.com
}

\section{Dear Editor,}

I would like to briefly discuss on "Actinic Keratosis - the probable cause of squamous cell skin carcinomas". Actinic keratosis is one of the most commonly existing skin lesions among the dermatological studies. It is estimated that it affects nearly $60 \%$ of US population and about $40-50 \%$ of Australian population above 40 years of age, who are chronically exposed to sunlight. Actinic keratosis is otherwise clinically referred to as solar keratosis, senile keratosis or simply as AK. It is considered as a precancerous skin lesion, leading to Squamous Cell Carcinomas (SCC) [1]. Most of the cutaneous squamous cell carcinomas are reported to be developed from the actinic keratosis. It is characterized by rough, scaly or crusty skin, with no specific signs or symptoms. One characteristic feature of actinic keratosis is that it resolves on its own, however, on exposure to UV rays, it once again reverts back. The biggest challenge to dermatologist is to predict which lesion would develop into a carcinoma. Hence, all actinic keratosis lesions are removed as a precautionary measure. AK usually affects face, scalp, lips, ears and hands. The clinical picture of AK shows a large size of lesion and ulceration, proving an increased risk of disease progression [2]. The risk becomes future complicated, if the patient shows signs of extensive solar damage, of advanced age and immunosuppression. Patients affected with actinic keratosis can be treated by lowering the duration of exposure to sun's ultraviolet rays. Nonetheless, different medications are also available to treat actinic keratosis. The first approved medicine was 5-fluorouracil, followed by Imiquimod 5\% and Diclofenac [3]. Very recently, a medication called Ingenol Mebulate (Picato), an active compound derived from a plant called Euphorbia peplus, is highly recommended [4]. It cures AK by inducing cell death. The advantage of using Ingenol Mebulate over others medications is because of its short duration of treatment. Ingenol Mebulate can be topically applied for 2-3 days depending on the affected area. Side effects include redness, skin irritation and sometimes swelling of the affected location. However, these side effects are an indication that the drug is working perfectly, and these side effects will weaken over a due course of few weeks.

In order to protect oneself from the risks of actinic keratosis, one need to reduce the exposure time in the sun, protecting the skin by applying sunscreen lotion with SPF $30+$ and wearing heat protective clothes. Regular visits to dermatologists for the general full body skin examination may to a certain extent prevent us from differentiating actinic keratosis and SCC.

\section{References}

1. Lalji A, Khiroya N, Lalji M. Actinic Keratosis and Squamous Cell Carcinoma. Clin Res Dermatol Open Access. 2014;1(1):1-3.

2. Rigel DS, Stein Gold LF. The importance of early diagnosis and treatment of actinic keratosis. J Am Acad Dermatol. 2013;68(1 Suppl 1):S20-27. doi: 10.1016/j.jaad.2012.10.001.

3. Christopher G Nelson. Diclofenac gel in the treatment of actinic keratoses. Therapeutics and Clinical Risk Management. 2011;7:207-211. doi: 10.2147/TCRM.S12498

4. Mazzeo M, Dattola A, Saraceno R, Bianchi L. A Case of Actinic Keratosis of the Scalp (Re)Treated With Ingenol Mebutate 150 Mcg/G. Clin Res Dermatol Open Access. 2016;3(5):1-2. 